\title{
Latent tuberculosis screening and treatment among asylum seekers: a mixed-methods study
}

\author{
Ineke Spruijt (10 ${ }^{1,2}$, Dawit Tesfay Haile ${ }^{2}$, Jeanine Suurmond ${ }^{2}$, Susan van den Hof ${ }^{1,3}$, \\ Marga Koenders ${ }^{4}$, Peter Kouw ${ }^{5}$, Natascha van Noort ${ }^{6}$, Sophie Toumanian ${ }^{7}$, \\ Frank Cobelens ${ }^{8}$, Simone Goosen ${ }^{9}$ and Connie Erkens ${ }^{1}$
}

@ERSpublications

LTBI screening and treatment among asylum seekers is feasible and effective when high quality of care is provided, including special attention for culture-sensitive education about TB and LTBI and collaboration with partner organisations http://bit.ly/2Lf6Y1I

Cite this article as: Spruijt I, Tesfay Haile D, Suurmond J, et al. Latent tuberculosis screening and treatment among asylum seekers: a mixed-methods study. Eur Respir J 2019; 54: 1900861 [https://doi.org/ 10.1183/13993003.00861-2019].

\section{ABSTRACT}

Introduction: Evidence on conditions for implementation of latent tuberculosis infection (LTBI) screening and treatment among asylum seekers is needed to inform tuberculosis (TB) control policies. We used mixed-methods to evaluate the implementation of an LTBI screening and treatment programme among asylum seekers in the Netherlands.

Methods: We offered voluntary LTBI screening to asylum seekers aged $\geqslant 12$ years living in asylum seeker centres from countries with a TB incidence $>200$ per 10000 population. We calculated LTBI screening and treatment cascade coverage, and assessed associated factors with Poisson regression using robust variance estimators. We interviewed TB care staff (seven group interviews) and Eritrean clients (21 group and 21 individual interviews) to identify programme enhancers and barriers.

Results: We screened 719 (63\% of 1136) clients for LTBI. LTBI was diagnosed among 178 (25\%) clients; 149 (84\%) initiated LTBI treatment, of whom 129 (87\%) completed treatment. In-person TB and LTBI education, the use of professional interpreters, and collaboration with partner organisations were enhancers for LTBI screening uptake. Demand-driven LTBI treatment support by TB nurses enhanced treatment completion. Factors complicating LTBI screening and treatment were having to travel to public health services, language barriers and moving from asylum seeker centres to the community during treatment.

Conclusion: LTBI screening and treatment of asylum seekers is feasible and effective when high quality of care is provided, including culture-sensitive TB education throughout the care cascade. Additionally, collaboration with partner organisations, such as agencies responsible for reception and support of asylum seekers, should be in place.

\footnotetext{
This article has supplementary material available from erj.ersjournals.com

Data availability: The data supporting the results of this study were obtained from multiple sources. We received permission from these sources to use the data for this study purpose, including publishing results of the study. However, no permission was sought to publish an anonymous dataset. Therefore, anonymous data supporting the study results can be made available only upon request, upon approval by the Dutch National Tuberculosis Registration Committee.
} 


\section{Introduction}

Asylum seekers from high tuberculosis (TB) incidence countries are at increased risk for developing TB after arrival in the host country due to high TB infection risk in the country of origin and during their journey to the host country, higher prevalence of risk factors for TB such as malnutrition and HIV, and more deprived living conditions in the host country [1]. In the Netherlands, this is reflected by the high incidences among Eritrean and Somalian asylum seekers and refugees in the first year after arrival (941 and 1107 per 10000 person-years, respectively) [2].

The Netherlands aimed to reduce TB incidence between 2016 and 2019 by $25 \%$ from 5.2 to 3.9 per 10000 population [3]. After a declining TB incidence trend in the Netherlands since 2009, TB incidence increased in 2015 and 2016 as a result of the high number of newly arriving asylum seekers [4]. Currently, asylum seekers from countries with a TB incidence $>50$ per 10000 are screened by chest radiography at arrival for TB disease. Those aged $>12$ years from countries with a TB incidence $>200$ per 10000 are offered additional 2-year biannual follow-up screening by chest radiography [5]. However, coverage of the follow-up screening among asylum seekers living in asylum seeker centres (ASCs) is suboptimal, fluctuating between $32 \%$ and $86 \%$ during 2009-2014 [6,7]. Because of suboptimal coverage and the fact that chest radiography screening only detects existing pulmonary TB disease, only $36 \%$ of TB patients among asylum seekers eligible for screening are detected through follow-up screening [5]. Chest radiography screening will thus unlikely be sufficient to achieve the envisioned decline in TB incidence. To reach pre-elimination, we need to focus on TB prevention, by screening and treating high TB risk groups such as asylum seekers for latent TB infection (LTBI).

LTBI screening and treatment has been considered more cost-effective than current chest radiography screening in the Netherlands [8]. TB policy advisors are therefore considering replacing the chest radiography follow-up screening with LTBI screening. However, evidence on conditions for effective implementation of LTBI screening and treatment among asylum seekers is limited. We used mixed-methods to study the feasibility and effectiveness of LTBI screening and treatment among asylum seekers in the Netherlands.

\section{Materials and methods}

\section{Study population and setting}

We invited asylum seekers eligible for chest radiography follow-up screening and living in an ASC (operated by the Central Agency for the Reception of Asylum Seekers (COA; www.coa.nl)) for TB symptom screening and LTBI testing from November 2016 until December 2017. Asylum seekers who had a history of TB disease or LTBI were not eligible for LTBI screening and were offered chest radiography screening.

\section{LTBI screening and treatment procedures}

Eight (out of 25) public health services (PHSs) organised and executed the LTBI screening, either at the PHS or at the ASC. The LTBI screening consisted of education, a health questionnaire (filled in by clients) and blood sampling. The PHSs used the QuantiFERON-TB Gold Plus (Qiagen, Germantown, MD, USA) interferon- $\gamma$ release assay (IGRA) according to the manufacturer's instructions for LTBI testing [9]. After exclusion of TB disease among participants with an IGRA result $\geqslant 0.35 \mathrm{IU} \cdot \mathrm{mL}^{-1}, \mathrm{~TB}$ physicians confirmed LTBI diagnosis and offered participants eligible for LTBI treatment 3-month isoniazid/rifampicin combination therapy free of charge. TB nurses provided LTBI treatment support based on the client's needs. PHSs followed recommendations of the Dutch guidelines for LTBI screening and treatment $[9,10]$. A detailed description of procedures throughout the LTBI screening and treatment cascade is given in supplementary file S1.

Affiliations: ${ }^{1} \mathrm{KNCV}$ Tuberculosis Foundation, The Hague, The Netherlands. ${ }^{2}$ Dept of Public Health, Amsterdam Public Health Research Institute, Amsterdam University Medical Centre, University of Amsterdam, Amsterdam, The Netherlands. ${ }^{3}$ National Institute for Public Health and the Environment (RIVM), Centre for Infectious Disease Control, Bilthoven, The Netherlands. ${ }^{4}$ Dept of Tuberculosis Control, Public Health Service Gelderland Zuid, Nijmegen, The Netherlands. ${ }^{5}$ Dept of Tuberculosis Control, Public Health Service Flevoland, Lelystad, The Netherlands. ${ }^{6}$ Dept of Tuberculosis Control, Public Health Service Region Utrecht, Utrecht, The Netherlands. ${ }^{7}$ Dept of Tuberculosis Control, Public Health Service Twente, Enschede, The Netherlands. ${ }^{8}$ Dept of Global Health and Amsterdam Institute for Global Health and Development, Amsterdam University Medical Center, Amsterdam, The Netherlands. ${ }^{9}$ Netherlands Association of Community Health Services (GGD GHOR Nederland), Utrecht, The Netherlands.

Correspondence: Ineke Spruijt, KNCV Tuberculosis Foundation, Benoordenhoutseweg 46, 2501 CC The Hague, The Netherlands. E-mail: ineke.spruijtakncvtbc.org 


\section{Data collection and analyses}

\section{Quantitative data}

We collected data from clients' health questionnaires including data on demographics and data from questionnaires (filled in by the TB physician) evaluating LTBI treatment, including reasons for not initiating or completing LTBI treatment. All questionnaire data were double entered in Microsoft Access (Microsoft, Seattle, WA, USA) and checked against the raw data for inconsistencies. We collected reasons for loss to follow-up and diagnostic and treatment data from the electronic patient registration system of the PHS and the National TB Registration system. We merged all datasets and approached the PHS for validation and substitution of missing data.

We calculated LTBI screening coverage (number screened/number invited) and proportions for each step of the LTBI screening and treatment cascade, and reported reasons for loss to follow-up and not initiating or completing LTBI treatment. Additionally, we assessed factors associated with LTBI diagnosis and LTBI treatment initiation and completion in all case regression analyses. As probabilities for outcome occurrence (LTBI diagnosis 25\%, LTBI treatment initiation $84 \%$ and LTBI treatment completion $87 \%$ ) were not rare, we fitted univariable Poisson regression models with robust variance estimators to calculate risk ratios [11]. We considered applying a random effects model to take into account clustering at the PHS level. However, numbers of individuals per PHS group were too small to produce valid estimates [12]. We therefore did not apply a random effects model, but fixed the PHS as a variable in the multivariable Poisson regression models to adjust for potential effects associated with clustering of PHSs. We checked for interaction between independent variables before fitting multivariable models. We used backward elimination of the initial model with variables yielding a $p$-value $<0.2$ in univariable analysis, guided by changes in regression coefficients and in the model fit. We recoded and analysed data using SPSS Statistics version 25.0 (IBM, Armonk, NY, USA).

\section{Qualitative data}

For our semistructured interviews, we designed topic guides based on Lévesque's conceptual framework, which distinguishes five dimensions in a systematic pathway for access to care: approachability, acceptability, availability and accommodation, affordability, and appropriateness (supplementary file S2) [13].

We interviewed TB care staff (seven group interviews) from each PHS and Eritrean clients (21 group interviews and 21 individual interviews). We interviewed only Eritrean clients as they were the largest group of asylum seekers in the ASCs eligible for LTBI screening during the study period. Table 1 provides a detailed overview of the qualitative research methodology.

We transcribed all audio-taped interviews. After familiarisation with the data, one of the authors (I. Spruijt) developed three coding schemes to guide the coding of transcripts from the interviews. In regular meetings, three authors (D. Tesfay Haile, I. Spruijt and J. Suurmond) discussed coding, categories and interpretation of the data [14]. We used MAXQDA version 11 (VERBI, Berlin, Germany) to assist in analyses of qualitative data.

\section{Ethics approval}

The Medical Ethical Committee of University Medical Centre Amsterdam (Amsterdam, the Netherlands) waived the need for ethical approval of this study because the Dutch Medical Research Involving Human Subjects Act does not apply given that the study was primarily focused on finding and treating TB, and PHSs are licensed to conduct screening for LTBI in the Netherlands. We followed the ethical principles of the Declaration of Helsinki.

\section{Results}

\section{Quantitative results}

The average coverage of LTBI screenings was $62 \%$. The coverage of LTBI screenings organised at the ASC (average 63\%; minimum 50\%, maximum 87\%) was slightly higher and fluctuated less than the coverage of LTBI screenings organised at the PHS (average 59\%; minimum 8\%, maximum 96\%) (supplementary file S3). In total, 719 clients completed LTBI screening, of whom the majority were male, aged 18-34 years, of Eritrean origin, had a low level of education and stayed alone in the Netherlands. The average stay of clients in the Netherlands at the time of LTBI screening was 11 months (table 2). Although population characteristics differed slightly between PHSs, they were comparable to the distribution of total population characteristics.

Of 719 clients screened for LTBI, 205 (29\%) had a positive IGRA result, of whom 20 (3\%) were lost to follow-up, 178 (25\%) were diagnosed with LTBI, four $(0.5 \%)$ had TB disease and three $(0.4 \%)$ had been treated for TB disease in the past. During the LTBI screening, 95 clients reported symptoms suggestive of TB disease on their health questionnaire. For unknown reasons, TB care staff did not follow protocol accordingly, as only $13 \%$ of those clients received a chest radiograph (figure 1 ). 
TABLE 1 Qualitative research methods

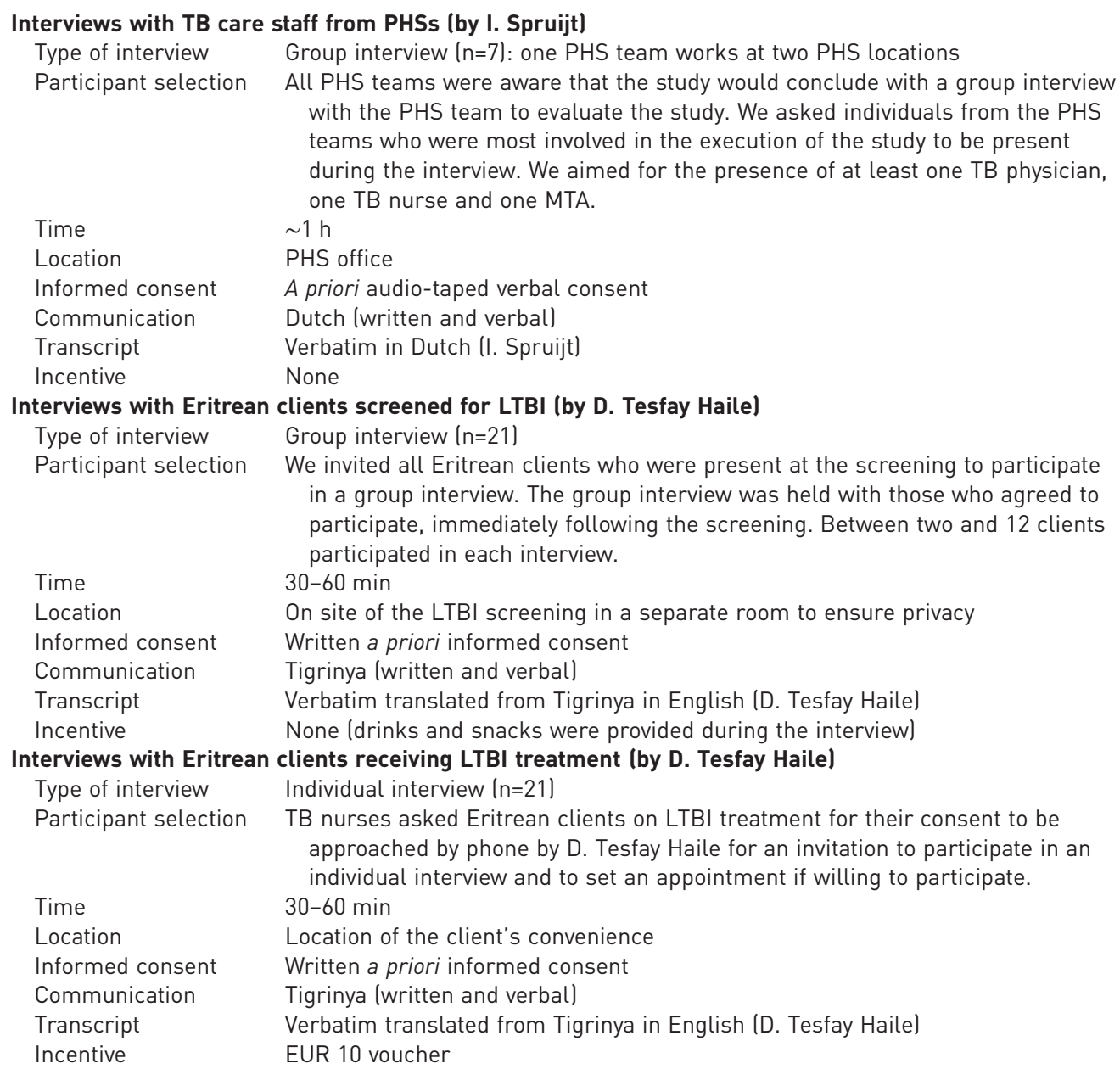

TB: tuberculosis; PHS: public health service; MTA: medical technical assistant; LTBI: latent TB infection.

In multivariable analysis, male clients and clients in older age groups had higher risk for LTBI diagnosis, whereas Eritrean clients and those with higher levels of education had lower risk for LTBI diagnosis (table 3).

149 (84\%) clients initiated LTBI treatment, of whom seven (5\%) were lost to follow-up, 13 discontinued treatment and 129 (87\%) completed LTBI treatment (figure 1). TB physicians reported side-effects among $50(34 \%)$ clients, of whom three (23\% of 13 clients) discontinued LTBI treatment (supplementary file S4). In multivariable analyses, Eritreans (versus non-Eritreans) were more likely to initiate LTBI treatment, whereas those in higher age groups (versus those aged $<24$ years) were less likely to start LTBI treatment (table 4).

\section{Qualitative results \\ Uptake of LTBI screening}

Although the uptake of LTBI screening organised at the ASC was only slightly higher, PHS staff identified several facilitators: 1) planning the screening in collaboration with the ASC-COA personnel during weekly mandatory reporting ensured the client's presence, 2) ASC-COA personnel could help locate clients in case of no-show, and 3) allowing clients to call and invite friends or family eligible for LTBI screening. A barrier for reaching high screening coverage, regardless of screening location, was the relocation of clients between ASCs or from the ASC to the municipalities.

TB nurse: "When we checked with the ASC (COA) personnel, we noticed that a lot of people who did not show up at the screening, did not live in the ASC anymore. ... More than half of them already moved." (Group interview 7, PHS) 
TABLE 2 Descriptive characteristics of clients throughout the latent tuberculosis infection (LTBI) screening and treatment programme

\begin{tabular}{|c|c|c|c|c|c|c|c|c|}
\hline & \multicolumn{2}{|c|}{ LTBI screening } & \multicolumn{2}{|c|}{ LTBI diagnosis } & \multicolumn{2}{|c|}{$\begin{array}{c}\text { Started LTBI } \\
\text { treatment }\end{array}$} & \multicolumn{2}{|c|}{$\begin{array}{c}\text { Completed LTBI } \\
\text { treatment }\end{array}$} \\
\hline & $\mathrm{n}$ & Column \% & $\mathrm{n}$ & Row \% & $\mathbf{n}$ & Row \% & $\mathbf{n}$ & Row \% \\
\hline \multicolumn{9}{|l|}{ PHS } \\
\hline 1 & 91 & 13 & 29 & 32 & 26 & 90 & 23 & 89 \\
\hline 2 & 99 & 14 & 26 & 26 & 23 & 89 & 21 & 91 \\
\hline 5 & 55 & 8 & 11 & 20 & 10 & 91 & 9 & 90 \\
\hline 6 & 51 & 7 & 19 & 37 & 15 & 79 & 13 & 87 \\
\hline 7 & 60 & 8 & 21 & 35 & 14 & 67 & 14 & 100 \\
\hline 8 & 198 & 28 & 32 & 16 & 26 & 81 & 21 & 81 \\
\hline \multicolumn{9}{|l|}{ Moved to different PHS region" } \\
\hline No & 573 & 80 & 105 & 18 & 90 & 86 & 74 & 82 \\
\hline Male & 414 & 58 & 117 & 28 & 101 & 86 & 87 & 86 \\
\hline \multicolumn{9}{|l|}{ Age category years } \\
\hline $0-17$ & 60 & 8 & 8 & 13 & 8 & 100 & 7 & 88 \\
\hline $18-24$ & 219 & 31 & 39 & 18 & 37 & 95 & 32 & 87 \\
\hline $25-34$ & 269 & 37 & 75 & 28 & 58 & 77 & 49 & 85 \\
\hline$\geqslant 35$ & 171 & 24 & 56 & 33 & 46 & 82 & 41 & 89 \\
\hline \multicolumn{9}{|l|}{ Country of origin } \\
\hline Non-Eritrea & 204 & 28 & 61 & 30 & 45 & 74 & 37 & 82 \\
\hline Eritrea/Ethiopia ${ }^{+}$ & 515 & 72 & 117 & 23 & 104 & 89 & 92 & 89 \\
\hline \multicolumn{9}{|l|}{ Household composition } \\
\hline Alone & 490 & 68 & 127 & 26 & 109 & 86 & 96 & 88 \\
\hline With family & 176 & 25 & 39 & 22 & 31 & 80 & 24 & 77 \\
\hline Missing & 53 & 7 & 12 & 23 & 9 & 75 & 9 & 100 \\
\hline$>6-\leqslant 12$ & 173 & 24 & 55 & 32 & 47 & 85 & 44 & 94 \\
\hline$>12$ & 211 & 30 & 75 & 35 & 68 & 91 & 60 & 88 \\
\hline Missing & 207 & 29 & 25 & 12 & 14 & 56 & 13 & 93 \\
\hline
\end{tabular}

PHS: public health service. " ${ }^{\text {: }}$ clients who moved (after the LTBI screening) to another asylum seeker centre or to a municipality in another PHS region; ": top five countries of origin other than Eritrea/Ethiopia: Somalia ( $n=43(6 \%))$, Pakistan (n=32 (5\%)), Nigeria ( $=31(4 \%))$, Uganda $(n=21(3 \%))$ and Democratic Republic of the Congo $(n=17(2 \%)){ }^{+}:$we combined Eritrea and Ethiopia as country of birth because many asylum seekers born in Ethiopia were of Eritrean nationality.

Another barrier for LTBI screening uptake was gossip in the ASC about the amount of blood collected for the blood test (respondents thought it was four bottles instead of four tubes). A few clients said this made them hesitative to go to the screening. However, most Eritrean clients perceived the screening as important for both their own health and public health, particularly as many perceived themselves at increased risk for infectious diseases (including TB) because of the communal living conditions in the ASC or because of their time spent in crowded unventilated transfer locations with poor hygiene, surrounded by many persons in poor health and during their journey to the Netherlands. They also expressed their need to be tested for other medical conditions, such as HIV and hepatitis.

Eritrean client: "Most of us crossed the Sahara. ... We survived by drinking any available water: sometimes water that you would not even dare to touch. We are still alive, but we never did any 


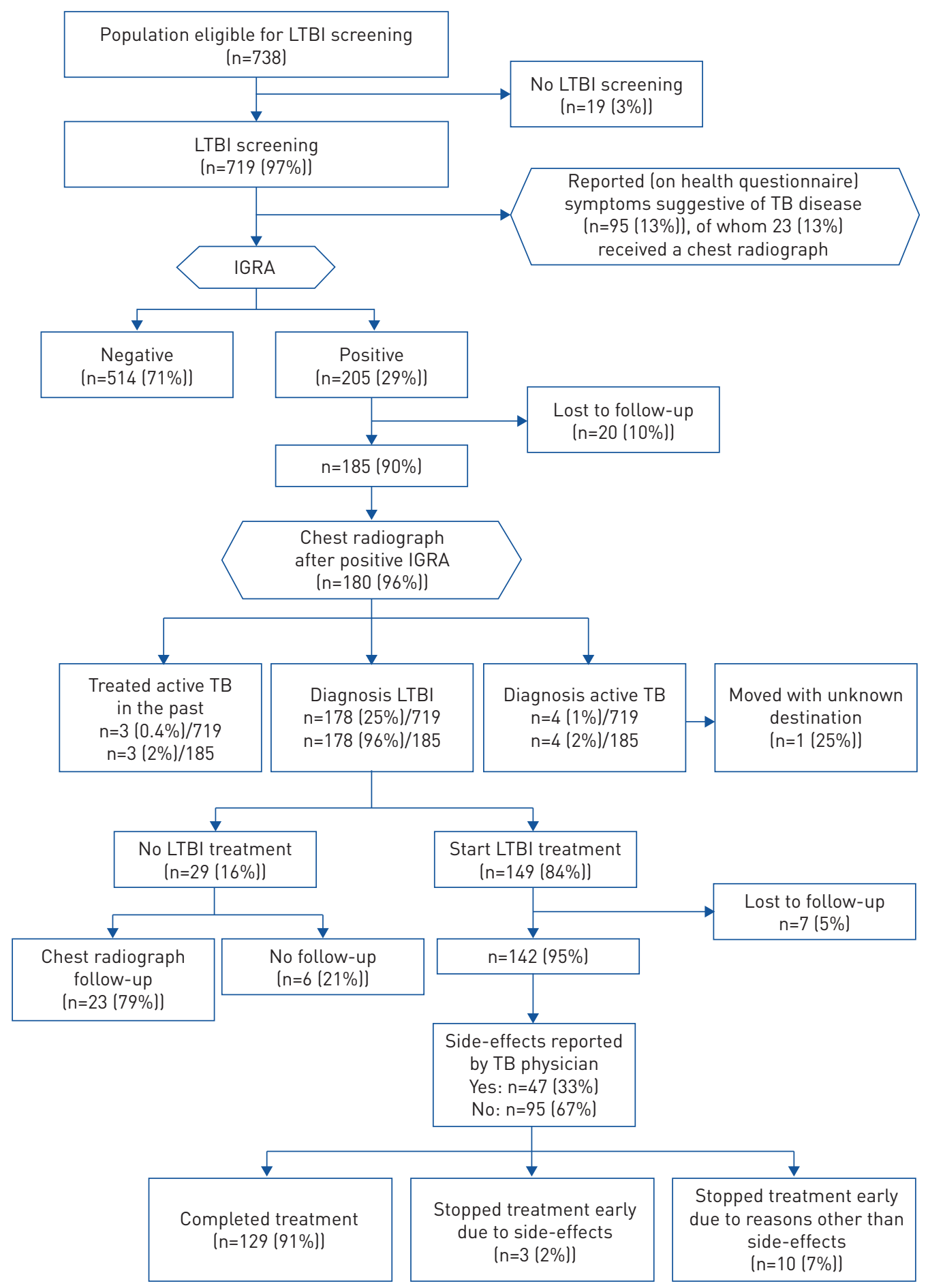

FIGURE 1 Flowchart of latent tuberculosis (TB) infection (LTBI) screening and treatment results. IGRA: interferon- $\gamma$ release assay.

medical examination. I would like to know if they could do overall check-ups, including the internal organs." (Group interview 5, clients)

Information and education

PHS staff said that most clients had a poor understanding of health-related topics and noted the clients' need for help to fill in the health questionnaire using a professional interpreter. However, many medical technical assistants (MTAs) doubted the feasibility of this individual approach in daily practice because of time constraints. Furthermore, some MTAs believed that information brochures, such as used for the 
TABLE 3 Results of univariable and multivariable Poisson regression models with robust variance estimators for client characteristics associated with latent tuberculosis infection (LTBI) diagnosis

\begin{tabular}{|c|c|c|c|c|c|c|}
\hline & \multicolumn{6}{|c|}{ LTBI diagnosis" } \\
\hline & \multicolumn{2}{|c|}{ Descriptive } & \multicolumn{2}{|c|}{ Unadjusted RR } & \multicolumn{2}{|c|}{ Adjusted RR ${ }^{\text {I }}$} \\
\hline & No & Yes & $\operatorname{RR}(95 \% \mathrm{CI})$ & p-value & $\operatorname{RR}(95 \% \mathrm{CI})$ & p-value \\
\hline Total & $514(75)$ & $168(25)$ & & & & \\
\hline \multicolumn{7}{|l|}{ Sex } \\
\hline Female & $237(80)$ & $59(20)$ & 1 & & 1 & \\
\hline Male & $277(72)$ & 109 (28) & $1.4(1.1-1.9)$ & 0.01 & $1.4(1.1-1.9)$ & 0.02 \\
\hline \multicolumn{7}{|l|}{ Age category years } \\
\hline $0-24$ & 218 (83) & $46(17)$ & 1 & & 1 & \\
\hline $25-34$ & $187(73)$ & $70(27)$ & $1.6(1.1-2.2)$ & 0.01 & $1.6(1.2-2.3)$ & 0.00 \\
\hline$\geqslant 35$ & 109 (68) & $52(32)$ & $1.9(1.3-2.6)$ & 0.00 & $1.7(1.2-2.3)$ & 0.00 \\
\hline \multicolumn{7}{|l|}{ Country of origin } \\
\hline Non-Eritrea & 135 (69) & $60(31)$ & 1 & & 1 & \\
\hline Eritrea/Ethiopia $^{+}$ & $379(78)$ & $108(22)$ & $0.7(0.6-0.9)$ & 0.02 & $0.8(0.6-1.0)$ & 0.07 \\
\hline \multicolumn{7}{|l|}{ Education level } \\
\hline No formal/primary education & $166(70)$ & $72(30)$ & 1 & & 1 & \\
\hline Secondary education & $248(80)$ & $64(20)$ & $0.7(0.5-0.9)$ & 0.01 & $0.7(0.6-1.0)$ & 0.04 \\
\hline Higher education & $100(76)$ & $32(24)$ & $0.8(0.6-1.2)$ & 0.23 & $0.7(0.5-1.1)$ & 0.09 \\
\hline \multicolumn{7}{|c|}{$\begin{array}{l}\text { Data are presented as } n(\%) \text {, unless otherwise stated. RR: risk ratio. }{ }^{\#} \text { : all case analyses: } 682(94.9 \%) \text { out } \\
\text { of } 719 \text { asylum seekers invited for screening; }{ }^{\eta}: \text { multivariable RRs are adjusted for public health service; } \\
{ }^{+}: \text {we combined Eritrea and Ethiopia as country of birth because many asylum seekers born in Ethiopia } \\
\text { were of Eritrean nationality. }\end{array}$} \\
\hline
\end{tabular}

current chest radiography screening, were sufficient and a verbal explanation with a professional interpreter was not necessary.

MTA: "When this [LTBI screening] becomes policy ... I don't think I will constantly explain everything in each consultation ... I also do not explain everything when people come for their chest X-ray screening." (Group interview 4, PHS)

Most clients, however, said they did not read the information brochure because of negligence, the perceived difficult content or illiteracy. The in-person verbal education about TB and LTBI prior to the blood sampling was therefore very much appreciated and consequently an important facilitator for the acceptance of screening. Furthermore, clients perceived the education as essential for informed decision making for participation in the screening. Some clients believed that if the education would have been organised a few days prior to the screening, more people would have shown up at their screening appointment because they would have understood, or otherwise could have shared among each other, the importance of the screening.

Eritrean client: "Some people did not show up: they thought the screening was not important. I also had no intention to go. I happened to be in the camp [ASC] because I had other appointments. If people had received the explanation in advance, I think they wouldn't have missed the opportunity." (Group interview 7, clients)

\section{LTBI treatment acceptance and completion}

In this study, we found high LTBI treatment initiation proportions (84\%). LTBI treatment initiation was higher among Eritrean than non-Eritrean asylum seekers (adjusted risk ratio 1.2 (95\% CI 1.0-1.4)). TB physicians believed that most Eritrean clients initiated LTBI treatment because they did not distinguish TB disease from LTBI. However, most Eritrean clients said that, despite difficulties to fully comprehend the concept of LTBI, they initiated LTBI treatment to kill the TB bacteria and prevent themselves from developing TB in the future. Both clients and TB physicians observed the tendency of clients to follow the TB physician's advice.

Eritrean client: “They said I had the virus in my body and medication can cure me. I said: "I don't feel any symptoms, but if you say I need treatment I am willing to do as you say". If someone 
TABLE 4 Results of univariable and multivariable Poisson regression models with robust variance estimators for client characteristics associated with latent tuberculosis infection (LTBI) treatment initiation and completion

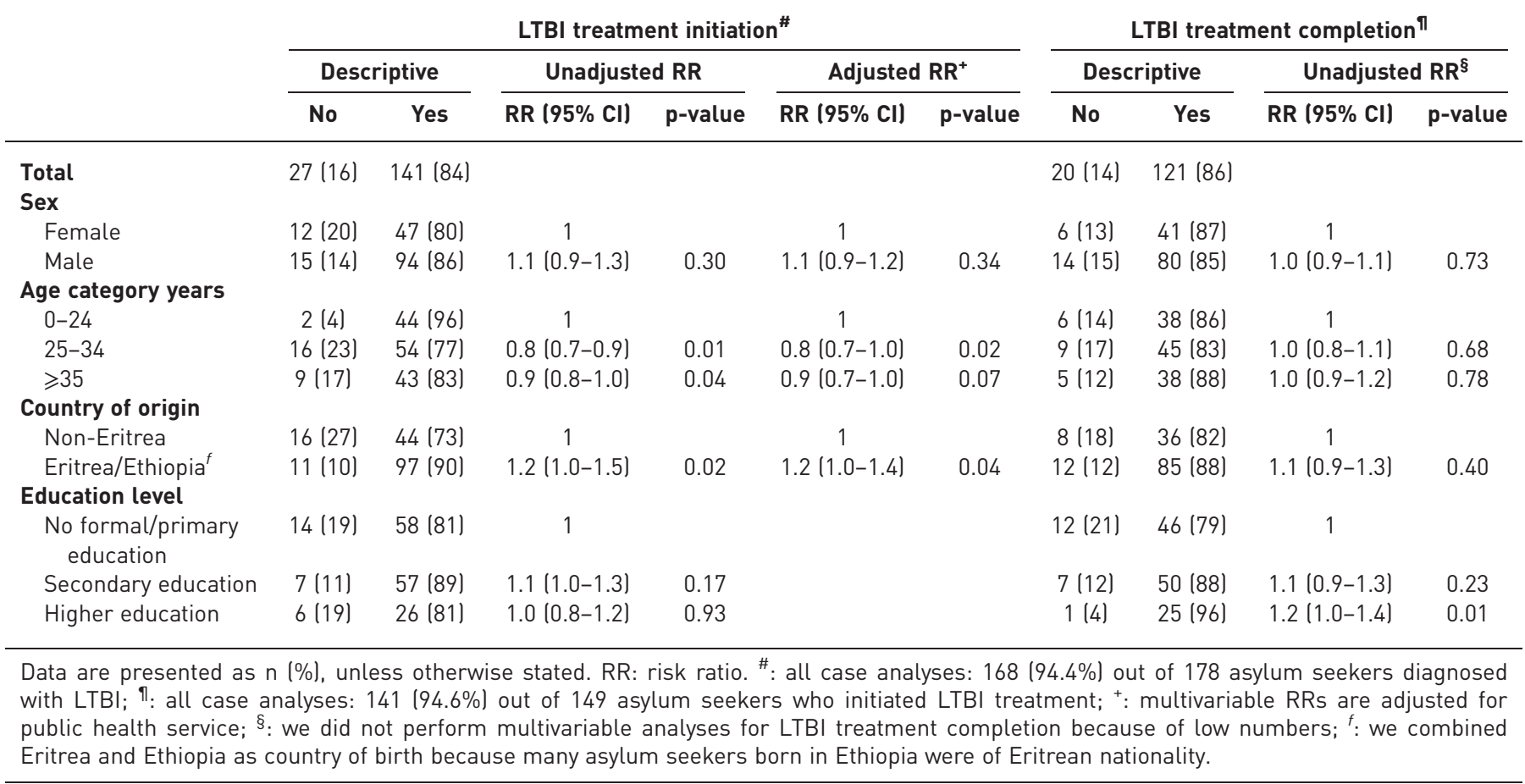

knows more than you, you should do as they say. Therefore, I accepted and took the treatment for 3 months without any problem." (Individual interview 15, clients)

PHS staff identified two major facilitators for high LTBI treatment completion (87\%): 1) the use of professional interpreters ( $81 \%$ of client consultations), which on the downside doubled consultation time, and 2) the LTBI treatment support given to $81 \%$ of clients by TB nurses, of which the intensity was comparable to that of TB disease treatment support (supplementary file S4).

TB physician: "They [asylum seekers] need more care and support. ... They are new in the Netherlands and they are facing so many challenges. ... I think, if you want to spend that extra attention and care, you can start treatment without hesitation." (Group interview 3, PHS)

Other facilitators for LTBI treatment continuation and completion were the use of alarms on mobile phones, WhatsApp contact between nurses and clients (using emoticons, spoken messages and very simple text messages), and week boxes for medication. Some clients who did not use week boxes felt overwhelmed with the number of tablets when given all at once for 1 or 2 months.

Eritrean client: "When I saw the medication for the first week it was OK: there were only 14 tablets. But when they gave me all the rest, I was shocked. I was not sure if I could complete my treatment."

(Individual interview 17, clients)

The relocation of clients from ASCs into communities was perceived as a challenge for treatment completion by both clients and TB nurses: clients experienced it as stressful and TB nurses experienced it as time consuming as they often had to assist in arranging practical issues, such as registration at pharmacies, to ensure continuity of care. Some TB physicians and some clients suggested to postpone the treatment until after relocation in the municipality.

TB physician: "It's not efficient to prescribe medication within this period: they don't know their address yet, they lose their medication, and it is difficult to arrange DOT [directly observed treatment] if indicated. The clients and I came to an agreement to postpone the treatment." (Group interview 2, PHS) 
A practical challenge for treatment continuation was clients not showing up (on time) for their appointments, which was the case for $15 \%$ of clients on LTBI treatment (supplementary file S4). Most clients said they did not know how to reschedule appointments and had financial or practical difficulties travelling to the PHS: they were unfamiliar with public transportation or (Google) maps, some were illiterate and dependent on the help of others to travel, and some had problems asking for help because of language barriers. Language was also a barrier for some clients experiencing side-effects: they could not reach out to PHS staff. A perceived enhancer by both clients and TB nurses was collaboration with the ASC health services. They could help filling the week boxes or function as a contact person for clients with queries concerning the treatment. Overall, most clients and PHS staff said in-person verbal explanation and demand-driven treatment support by TB nurses were essential for successful LTBI screening and treatment.

TB nurse practitioner: "It is feasible if you are willing to invest. If you give in-person verbal explanation, clients understand why they are screened and treated: they know it is for their own health. Do not make it too complicated: clients should not drop out because it takes them 2 hours to get to the PHS. You should find a balance: it is personalised medicine." (Group interview 6, PHS)

\section{Discussion}

We evaluated an LTBI screening and treatment programme among asylum seekers living in ASCs in the Netherlands, and identified barriers and enhancers for its implementation. High LTBI screening and treatment uptake and completion can be reached if in-person verbal education on TB and LTBI is given, professional interpreters are used, and demand-driven LTBI treatment support is provided. Furthermore, we showed that the programme is successful in identifying both LTBI and TB disease. However, we found that the study protocol requiring additional chest radiography screening of those with TB symptoms was not consistently followed, which could have led to undesirable delay in the diagnosis of TB disease in case of a false-negative IGRA. Digital tools (e.g. text messaging using emoticons) and collaboration with health services in the ASC helped to overcome persisting barriers related to language and transport, and therefore optimised LTBI treatment support.

LTBI treatment initiation and completion proportions were high, which is in contrast to a pilot study among immigrants [15]. Studies performed in other countries and settings also showed that these high proportions can be achieved among persons seeking asylum, provided that personalised LTBI treatment support by trained (TB) care staff, including education throughout the treatment process, is given [16-19]. Furthermore, the use of professional interpreters and peer educators is crucial to allow informed decision making of clients throughout the LTBI screening and treatment process, and these are important facilitators in LTBI treatment completion [20-23].

In practice, information provision is a somewhat neglected part of TB screening [24]. Furthermore, MTAs are under a time constraint to screen one person with chest radiography every 5-6 min. This time constraint will not allow MTAs to provide education, evaluate the health questionnaire and sample blood, which are required components of high-quality LTBI screening. Furthermore, using professional interpreters increases consultation time throughout the cascade. Time constraints thus hamper implementation of education activities and the use of professional interpreters, and consequently endanger LTBI screening and treatment uptake and completion. Competency training of all TB care staff and commitment to provide education and use professional interpreters when necessary, including the commitment of management, are therefore required to ensure a successful LTBI screening programme.

For optimal prevention of TB cases among asylum seekers, it is preferable to screen and treat for LTBI as soon as possible after entry. However, this is not feasible because of practical reasons related to asylum procedures and processes. Additionally, it would be unethical to screen those asylum seekers (69\% of all asylum seekers with first asylum application in 2017 [25]) who are unable to finish LTBI treatment because of their rejected asylum application and consequent deportation. A complicating factor for follow-up of clients is the mobility of asylum seekers: they move between ASCs or move from ASCs to municipalities $[26,27]$. Furthermore, fragmentation of the healthcare system can endanger continuation of care [28]. A major enhancer for continuation of care in our study was the willingness of most clients to be screened and treated for TB and LTBI. Clients also expressed their wish to be tested for other (infectious) diseases. Future screening programmes should seek the opportunity to collaborate with other public health departments to address unmet health needs of clients through a sustainable, integrated approach [27, 29-31].

A limitation of our study is that we interviewed only Eritrean clients. We therefore may have missed impeding and enhancing factors for LTBI screening and treatment experienced by clients of other nationalities. However, important barriers identified in this study, such as language, travelling and mobility, are likely to be generalisable to other nationalities. Furthermore, because of small sample sizes we 
could not stratify our analyses by Eritrean versus non-Eritrean nationality or apply a random effects model, nor could we conduct multivariable analyses for treatment completion. Those analyses would have led to overfitting of data and sparse data bias, and consequently produce estimates not representative of the true effect $[32,33]$.

In this study, we targeted persons from countries with a TB incidence $>200$ per 10000 population for LTBI screening. Previous studies among immigrants showed similar LTBI prevalence among those from countries with a TB incidence of 101-200 per 10000 as for those from countries with a TB incidence of $>200$ per $10000[15,34]$. This suggests that extending the target group for LTBI screening to asylum seekers from countries with an incidence $\leqslant 200$ per 10000 may have a larger impact on TB incidence. We are currently employing mathematical models to assess for which subgroups of asylum seekers (based on age and TB incidence in the country of origin and in the host country) LTBI screening and treatment programmes would be most cost-effective and would have the highest impact on TB incidences.

\section{Conclusions}

LTBI screening and treatment among asylum seekers living in ASCs is feasible. Essential components of successful implementation are the use of professional interpreters, in-person verbal education about TB and LTBI throughout the cascade of screening and care, collaboration with partner organisations, and LTBI treatment support. PHS staff should be trained, stimulated and allocated time to educate clients on TB and LTBI using professional interpreters. Our results will support future policy decision making on implementation of LTBI screening and treatment among asylum seekers from high TB incidence countries, and may also be valuable for other low TB incidence countries.

Acknowledgements: We would like to thank all the Netherlands PHS staff members, in particular Marja Karels and Maaike Heijnen (Dept of Tuberculosis Control, PHS Hollands Noorden), and Mirjam Urban, Annet Bak and Jolijn de Vries (Dept of Tuberculosis Control, PHS Groningen and Drenthe) for their dedicated work in TB control, especially for their work with the LTBI screening and treatment which enabled this study. We would like to thank the Central Agency for the Reception of Asylum Seekers (COA; The Hague, The Netherlands) for their in-kind contribution to reimburse LTBI tests and treatment. We would like to thank Agata Hinc from the COA and the COA and medical personnel working in the asylum seeker centres for their collaboration.

Author contributions: I. Spruijt wrote the study protocol in collaboration with the other authors, performed data management and analyses, and wrote the manuscript. D. Tesfay Haile conducted and transcribed interviews with Eritrean clients, and supported in qualitative data analyses and interpretation. C. Erkens was daily supervisor of I. Spruijt and approved the study protocol. J. Suurmond supervised the qualitative research of this study. S. van den Hof supervised the epidemiological component of this study and approved the study protocol. F. Cobelens provided input on all aspects of the study process. M. Koenders, P. Kouw, N. van Noort and S. Toumanian were coordinators of the study at their PHS. S. Goosen advised on the study process. All authors read, commented on and approved the final manuscript.

Conflict of interest: I. Spruijt reports grants and other from KNCV Tuberculosis Foundation, during the conduct of the study. D. Tesfay Haile has nothing to disclose. J. Suurmond has nothing to disclose. S. van den Hof has nothing to disclose. M. Koenders has nothing to disclose. P. Kouw has nothing to disclose. N. van Noort has nothing to disclose. S. Toumanian has nothing to disclose. F. Cobelens has nothing to disclose. S. Goosen has nothing to disclose. C. Erkens has nothing to disclose.

Support statement: This work was supported by the Netherlands Organisation for Health Research and Development (ZonMW; grant 50-53000-98-128). Qiagen provided the QuantiFERON-TB Gold Plus kits free of charge. Qiagen read the final manuscript. Neither ZonMW nor Qiagen had a role in the study design, data collection and analysis, decision to publish or preparation of the manuscript. Funding information for this article has been deposited with the Crossref Funder Registry.

\section{References}

1 Dobler CC, Fox GJ, Douglas P, et al. Screening for tuberculosis in migrants and visitors from high-incidence settings: present and future perspectives. Eur Respir J 2018; 52: 1800591.

2 van den Boogaard J, Slump E, Schimmel HJ, et al. Hoge TBC-incidentie bij Eritrese en Somalische asielzoekers in de eerste vijf jaar na aankomst. [High incidence of active tuberculosis in Eritrean and Somalian asylum seekers in the first five years after arrival in the Netherlands: time for a screening programme for latent infection.] Bilthoven, Rijksinstituut voor Volksgezondheid en Milieu, 2018.

3 de Vries G, Riesmeijer R. Nationaal Plan Tuberculosebestrijding 2016-2020. Op weg naar eliminatie. [National Plan TB Control 2016-2020. Towards elimination.] Bilthoven, Rijksinstituut voor Volksgezondheid en Milieu, 2016.

4 Slump E, Bregman IM, Erkens CGM, et al. Tuberculose in Nederland 2016. Surveillancerapport. [Tuberculosis in the Netherlands 2016. Surveillance Report.] Bilthoven, Rijksinstituut voor Volksgezondheid en Milieu, 2016.

5 van Rest J, Erkens CGM, De Vries G. Evaluatie tuberculosescreening asielzoekers, 2011-2015. [Evaluation of tuberculosis screening among asylum seekers in the Netherlands, 2011-2015.] The Hague, KNCV Tuberculosefonds, 2017. 
6 Netherlands Association of Community Health Services (GGD GHOR Nederlands). Analyse van de dekkingsgraad van TBC-vervolgscreeningen bij asielzoekers aangekomen in de jaren 2009-2011. [Analyses of the coverages of the TB follow-up screening among asylum seekers in 2009-2011.] Utrecht, GGD GHOR, 2013.

7 Van Rest J, Goosen S, ven Burg J, et al. Voorlopige evaluatie tuberculosescreening asielzoekers, 2011-2015. [Provisional evaluation of the follow-up screening among asylum seekers, 2011-2015.] The Hague, KNCV Tuberculosefonds, 2016.

8 European Centre for Disease Prevention and Control. Cost-effectiveness Analysis of Programmatic Screening Strategies for Latent Tuberculosis Infection in the EU/EEA. Stockholm, ECDC, 2018.

9 Commission Practical Tuberculosis Control. Richtlijn interferon gamma release assays bij de diagnostiek van tuberculose. [Guideline: interferon gamma release assay for the diagnosis of tuberculosis.] The Hague, KNCV Tuberculosefonds, 2010.

10 Commission Practical Tuberculosis Control. Richtlijn behandeling latente tuberculose-infectie. [Guideline: treatment of latent tuberculosis infection.] The Hague, KNCV Tuberculosefonds, 2015.

11 Knol MJ, Le Cessie S, Algra A, et al. Overestimation of risk ratios by odds ratios in trials and cohort studies: alternatives to logistic regression. CMAJ 2012; 184: 895-899.

12 Moineddin R, Matheson FI, Glazier RH. A simulation study of sample size for multilevel logistic regression models. BMC Med Res Methodol 2007; 7: 34.

13 Levesque JF, Harris MF, Russell G. Patient-centred access to health care: conceptualising access at the interface of health systems and populations. Int J Equity Health 2013; 12: 18.

14 Miles MB, Huberman M, Saldana J. Qualitative Data Analysis: A Methods Sourcebook. 3rd Edn. Thousand Oaks, Sage, 2014

15 Spruijt I, Erkens C, Suurmond J, et al. Implementation of latent tuberculosis infection screening and treatment among newly arriving immigrants in the Netherlands: a mixed methods pilot evaluation. PLoS One 2019; 14: e0219252.

16 Benjumea-Bedoya D, Becker M, Haworth-Brockman M, et al. Integrated care for latent tuberculosis infection (LTBI) at a primary health care facility for refugees in Winnipeg, Canada: a mixed-methods evaluation. Front Public Health 2019; 7: 57.

17 Olsson $\mathrm{O}$, Winqvist $\mathrm{N}$, Olsson $\mathrm{M}$, et al. High rate of latent tuberculosis treatment completion in immigrants seeking asylum in Sweden. Infect Dis 2018; 50: 678-686.

18 Schein YL, Madebo T, Andersen HE, et al. Treatment completion for latent tuberculosis infection in Norway: a prospective cohort study. BMC Infect Dis 2018; 18: 587.

19 Thee S, Kruger R, von Bernuth $\mathrm{H}$, et al. Screening and treatment for tuberculosis in a cohort of unaccompanied minor refugees in Berlin, Germany. PLoS One 2019; 14: e0216234.

20 Abarca Tomas B, Pell C, Bueno Cavanillas A, et al. Tuberculosis in migrant populations. A systematic review of the qualitative literature. PLoS One 2013; 8: e82440.

21 Butcher K, Biggs BA, Leder K, et al. Understanding of latent tuberculosis, its treatment and treatment side effects in immigrant and refugee patients. BMC Res Notes 2013; 6: 342.

22 Wieland ML, Weis JA, Yawn BP, et al. Perceptions of tuberculosis among immigrants and refugees at an adult education center: a community-based participatory research approach. J Immigr Minor Health 2012; 14: 14-22.

23 Jonzon R, Lindkvist P, Johansson E. A state of limbo - in transition between two contexts: Health assessments upon arrival in Sweden as perceived by former Eritrean asylum seekers. Scand J Public Health 2015; 43: 548-558

24 Dutch Health Council. WBO: tuberculose screening. [WBO: tuberculosis screening.] The Hague, Dutch Health Council, 2017.

25 Government of the Netherlands. Kerncijfers asiel en migratie, December 2017. [Key numbers asylum and migration, December 2017.] The Hague, Government of the Netherlands, 2018.

26 Alsdurf H, Hill PC, Matteelli A, et al. The cascade of care in diagnosis and treatment of latent tuberculosis infection: a systematic review and meta-analysis. Lancet Infect Dis 2016; 16: 1269-1278.

27 Seedat F, Hargreaves S, Nellums LB, et al. How effective are approaches to migrant screening for infectious diseases in Europe? A systematic review. Lancet Infect Dis 2018; 18: e259-e271.

28 Pontarelli A, Marchese V, Scolari C, et al. Screening for active and latent tuberculosis among asylum seekers in Italy: a retrospective cohort analysis. Travel Med Infect Dis 2019; 27: 39-45.

29 Delilovic S, Kulane A, Asbring N, et al. What value for whom? - provider perspectives on health examinations for asylum seekers in Stockholm, Sweden. BMC Health Serv Res 2018; 18: 601.

30 European Centre for Disease Prevention and Control. Infectious Diseases of Specific Relevance to Newly-arrived Migrants in the EU/EEA. Stockholm, ECDC, 2015.

31 Suurmond J, Rupp I, Seeleman C, et al. The first contacts between healthcare providers and newly-arrived asylum seekers: a qualitative study about which issues need to be addressed. Public Health 2013; 127: 668-673

32 Babyak MA. What you see may not be what you get: a brief, nontechnical introduction to overfitting in regression type models. Psychosom Med 2014; 66: 411-421.

33 Greenland S, Mansournia MA, Altman DG. Sparse data bias: a problem hiding in plain sight. BMJ 2016; 352 : i1981.

34 Pareek M, Watson JP, Ormerod LP, et al. Screening of immigrants in the UK for imported latent tuberculosis: a multicentre cohort study and cost-effectiveness analysis. Lancet Infect Dis 2011; 11: 435-444. 